\title{
Special Section Guest Editorial: Advances in Urban Imaging and Applications
}

\author{
Xiaohui Yuan, ${ }^{a}$ Sos Agaian, ${ }^{b}$ Wencheng Wang, ${ }^{c}$ and Mohamed Elhoseny ${ }^{d}$ \\ ${ }^{a}$ University of North Texas, United States \\ ${ }^{\mathrm{b}}$ CUNY, College of Staten Island, United States \\ ${ }^{\mathrm{c}}$ Weifang University, China \\ ${ }^{\mathrm{d}}$ Mansoura University, Egypt
}

Nowadays, a new generation of imaging, depth, and ultrasonic sensors and platforms is embedded in modern-day cities' infrastructures. Massive volume data from surveillance cameras, social media, and remote/proximate sensing platforms are currently harvested and stored that scaffold the transform of urban life. Using such rich data to support intelligent and efficient urban affairs management is a pressing demand to improve livability and accessibility for our citizens. The rapid growth of cities and the associated human activities lead to various urban problems, such as congestion, imbalanced resource allocation, and pollution. Urban imaging's interest increases day by day, especially since the COVID-19 pandemic and current world economic situation. On the other hand, the rapid advance of computing techniques for learning from massive data has profoundly changed our world in many aspects that significantly improve the performance of analysis of big data from our daily lives. Yet, the development and application of advanced computing methods for big urban data (text, images, and videos) analysis and management are limited, and intelligent technologies are still finding their feet in the fields of smart communities. New ideas that leverage the vast amount of diverse data from numerous sources and cutting-edge computing power to address emerging needs are urgently sought. In addition, urban intelligence leverages advanced sensing and network infrastructure and focuses on advancing algorithms and applications in artificial intelligence and data sciences to revolutionize our cities and communities. It serves as the underpinning driving force to the development and implementation of smart cities. It also integrates the science and technology transformation in computer sciences, engineering, health, transportation, energy, public safety, etc. The world population is growing, and it is estimated to double by 2050 . Therefore, these expectations produce new challenges and opportunities for cities and communities and urban imaging and its applications.

This Special Section on Advances in Urban Imaging and Applications presents the latest research in urban intelligence fields, emphasizing imaging and applications The International Conference on Urban Intelligence and Applications 2020 hosted presentations of many innovative ideas and new applications. Out of many papers recommended by the conference committee, six are accepted and included in this special section after thorough reviews and revisions. The included papers cover exciting topics of urban imaging, including novel algorithms and applications.

Maharjan et al. present a probabilistic non-rigid point set registration method to deal with large and uneven deformations, an ill-posed and highly challenging problem. By enforcing landmark correspondences, the proposed method preserves the point set's global shape with significant deformations. In addition, the stochastic neighbor embedding is used to protect local neighborhood structure, which penalizes incoherent transformation within a neighborhood.

Zhong et al. propose a fast and rotation-robust local difference binary descriptor using polar location. The method is based on a binary test of average intensity, radial gradient, and tangent gradient of grid cells on multiple log-polar grids. The computational cost is significantly reduced using a lookup table mapping discrete polar coordinates with image pixel locations and rebuilding the integral image in polar coordinates.

Fan et al. devise a track recognition algorithm based on a semi-supervised generative adversarial network that learns a robust model from a few examples distorted by outliers. The method

(ㄱ) 2021 SPIE and IS\&T 
identifies and eliminates the outliers and extracts strong flight features for deep network-based model creation.

Zhong et al. propose a facial expression recognition method based on the facial part attention that extracts emotional rich, local features. The proposed method leverages a cluster-based facial landmarks selection method and a convolution neural network. The convolutional neural network includes an object network for extracting facial features and an attention network for obtaining local features. The global and local features are integrated into the facial expression recognition model.

$\mathrm{Xu}$ et al. investigate the active balancing control with an aim towards real-time management of imaging platforms in response to external events. The method circumvents the inevitable delay in the high-speed systems. The method introduces an integral transformation term to convert a time-delay system into a dynamic model without delaying active balancing control.

Wang et al. study the synchronization of virtual camera motion attitude for improved accuracy in the multiplayer motion capture and virtual augmentation. The proposed system leverages the Unity3D platform and optical positioning technology to locate the position of the camera and virtual roles. The inertial attitude sensing technology is used to obtain multiple targets' attitude, which achieves an integration of the optical positioning and the inertial attitude sensor.

Xiaohui Yuan is an associate professor in the Department of Computer Science and Engineering, University of North Texas. His research interests include computer vision, data mining, machine learning, and artificial intelligence. His research is supported by Air Force Lab, National Science Foundation, Texas Advanced Research Program, Oak Ridge Associated Universities, and UNT. His research findings are reported in more than 180 peer-reviewed papers. He is a recipient of the Ralph E. Powe Junior Faculty Enhancement award in 2008 and the Air Force Faculty Fellowship in 2011, 2012, and 2013. He also received two research awards and a teaching award from UNT in 2007, 2008, and 2012, respectively. He served on the editorial board of several international journals and guest-edited several special issues. He is the general chair, program committee chair, and session chair in many conferences. He also serves as a panel reviewer for funding agencies, including NSF, NASA, NIH, and Louisiana Board of Regent's Research Competitiveness program.

Sos Agaian is a distinguished professor of Computer Science at the Graduate Center/CSI, CUNY, and was a Peter T. Flawn Professor of the University of Texas at San Antonio. His research interests include computational vision and machine learning, large-scale data analytic, multimodal data fusion, urban computing, biologically-inspired image processing modeling, multimodal biometric and digital forensics, 3D imaging sensors and security, and biomedical and health informatics. He has authored over 750 peer-reviewed research papers, 10 books, and 19 edited proceedings. He holds over 52 patents/disclosers. He is a fellow of IS\&T, SPIE, AAAS, IEEE, and a foreign member of the Armenian National Academy. He received the MAEStro Educator of the Year, sponsored by the Society of Mexican American Engineers, and the Distinguished Research Award at the University of Texas at San Antonio. He is the recipient of the Innovator of the Year Award (2014), of the Tech Flash Titans-Top Researcher-Award (San Antonio Business Journal, 2014), the Entrepreneurship Award (UTSA-2013 and 2016), and the Excellence in Teaching Award (2015). He is an associate editor for several journals and an editorial board member for the Journal of Pattern Recognition and Image Analysis and an associate editor for several journals, including the IEEE Transactions on Image Processing, the IEEE Transactions on Systems, Man and Cybernetics, Journal of Electrical and Computer Engineering, International Journal of Digital Multimedia Broadcasting and Journal of Electronic Imaging.

Wencheng Wang is currently a professor at Weifang University, Shandong, China. He engaged in the research of image dehazing and enhancement technology. He is also a principal investigator with the Weifang City's Key Laboratory and the Innovation Team of Shandong Provincial Education Department on robot's visual perception and control. He has published more than 60 articles in peer-reviewed academic journals and conferences. He holds over 14 patents. His main research interests include computer vision, pattern recognition, and intelligent computing. 
Mohamed Elhoseny is an associate professor at the Department of Computer Science, American University in the Emirates, Dubai, UAE. He is an ACM Distinguished Speaker and IEEE senior member. Collectively, he authored/co-authored over 150 published articles in prestigious journals. He authored/edited 17 international books published by recognized publishers such as Springer and Elsevier. He is the EiC of IJSSTA journal published by IGI Global and an associate editor at IEEE Journal of Biomedical and Health Informatics, IEEE Access, Scientific Reports, IEEE Future Directions, Remote Sensing, and International Journal of E-services and Mobile Applications. He is the editor-in-chief of the Studies in Distributed Intelligence Springer Book Series, the Sensors Communication for Urban Intelligence CRC Press-Taylor \& Francis Book Series, and the Distributed Sensing and Intelligent Systems CRC Press-Taylor \& Francis Book Series. 